\title{
Le monde politique vaudois au temps de Pareto à Lausanne
}

\section{Olivier Meuwly}

\section{OpenEdition}

\section{Journals}

Édition électronique

URL : http://journals.openedition.org/ress/750

DOI : 10.4000/ress.750

ISSN : 1663-4446

Éditeur

Librairie Droz

Édition imprimée

Date de publication : 1 juillet 2010

Pagination : 19-31

ISBN : 978-2-600-01449-6

ISSN : 0048-8046

\section{Référence électronique}

Olivier Meuwly, "Le monde politique vaudois au temps de Pareto à Lausanne », Revue européenne des sciences sociales [En ligne], XLVIII-146 | 2010, mis en ligne le 01 juillet 2013, consulté le 04 mai 2019.

URL : http://journals.openedition.org/ress/750 ; DOI : 10.4000/ress.750 
Olivier MEUWLY

\section{LE MONDE POLITIQUE VAUDOIS AU TEMPS DE PARETO À LAUSANNE}

\section{PARETO ENTRE LIBÉRAUX ET RADICAUX}

Contrairement à Léon Walras, Vilfredo Pareto n'a entretenu que peu de rapports avec le monde politique vaudois. Alors que Walras participait régulièrement aux dîners organisés par les chefs de file du pouvoir radical, les Ruchonnet, les Ruffy, Pareto limite son commerce avec les personnalités au pouvoir à son strict champ d'activité: la place de son enseignement à l'Université, ses appointements. Pour le reste, on sait qu'il a de bonnes relations avec quelques politiciens surtout libéraux, dont Charles Boiceau ${ }^{1}$. En revanche, à la différence de Walras, qui n'est consulté qu'occasionnellement par les plus hautes instances du pays, Pareto se mêle souvent de politique locale ou fédérale à travers certains thèmes sur lesquels on sollicite son avis, comme des expertises livrés aux autorités communales. Et la Gazette de Lausanne recueille souvent ses déterminations à la fois éclairées et militantes sur certains sujets d'actualité fédérale, comme la question de la Banque d'Etat, et quand bien même il reste fort prudent sur la vie politique de sa terre d'accueil.

Pareto n'en observait pas mois attentivement l'évolution politique de sa terre d'accueil. Faut-il pourtant donner crédit à l'affirmation de Georges Sorel selon laquelle Pareto, obtus à l'idée de lutte des classes, en réalité, «esp(érait) voir la société future s'inspirer des principes démocratiques du canton de Vaud $»^{2}$ ? Pareto, qui, lorsqu'il arrive à Lausanne en 1893, a conservé sa fougue très libérale et vise encore l'établissement d'un Etat minimal', se sent plutôt proche des

1 Né en 1841 et décédé en 1907, Boiceau, fils d'un banquier proche de Ruchonnet, avocat de profession, est conseiller communal à Lausanne de 1871 à 1874 et de 1888 à 1905; il est élu au Grand Conseil en 1874, avant d'accéder immédiatement au Conseil d'Etat où il siège jusqu'en 1885, date à laquelle il retourne au Grand Conseil. Conseiller national de 1878 à 1881 et de 1893 à 1899, il est administrateur de nombreuses et importantes sociétés. Il avait succédé au Conseil d'Etat à Ruchonnet, parvenant à briser pour quelques années l'hégémonie radicale: modéré, ayant aussi profité, en la circonstance, d'un relâchement de la discipline radicale au Parlement cantonal, il s'était également attiré maintes sympathies chez ses adversaires en réglant avec élégance un scandale dans lequel l'administration radicale avait été accusée d'avoir pris le parti d'étudiants contre la prétendue sévérité de certains professeurs.

2 Georges Sorel, Le devenir social, mai 1896, p. 468, note 1, cité par Giovanni Busino, Introduction à une histoire de la sociologie de Pareto, Genève, Droz, 1968, p. 151. Je remercie M. Fiorenzo Mornati des nombreuses informations bio- et bibliographiques qu'il a bien voulu me transmettre.

3 Philippe Steiner, Vilfredo Pareto et la révision du libéralisme classique, in Philippe Nemo et Jean Petitot, Histoire du libéralisme en Europe, Paris, Presses universitaires de France, 2006, pp. 595-618. 
responsables du parti libéral-conservateur vaudois. Et il ne fait aucun doute que radicaux et Pareto se regardent avec une certaine distance. A l'annonce de la nomination du marquis italien, la Revue, journal des radicaux vaudois, s'en félicite, mais sans s'étendre très longuement sur l'événement. Le quotidien dirigé par Félix Bonjour note l'arrivé d'un homme «très connu et très apprécié », avant de souligner ce «nouveau succès» de «notre jeune Université» ${ }^{4}$.

Et c'est avec curiosité que la feuille radicale assiste à la première conférence académique donnée par l'enseignant italien. En février 1894, Pareto défend une vision extrêmement restrictive de l'idée d'Etat social, qui commence à se développer dans le monde politique radical, et aurait plaidé pour les vertus de la charité privée, comme fondement d'une politique sociale authentique ${ }^{5}$. La charité prônée par l'ingénieur économiste, reconnaît la Revue, n'a certes rien à voir avec une «charité vulgaire». Selon l'orateur, et aux dires du journal radical, la lutte contre l'impôt et le renchérissement de l'existence aurait des effets bien plus bénéfiques que toute politique d'assistance pilotée par l'Etat. L'organe radical prend néanmoins ses distances des allégations du savant transalpin. La Revue admet l'habileté du conférencier mais ajoute tout de go que ce dernier ne «nous a pas converti ». Pour les radicaux, l'assistance officielle est bel et bien une nécessité aujourd'hui. Seuls les contours à donner à cette assistance officielle posent problème et traceront la ligne de partage qui se creuse désormais irrémédiablement entre radicaux et socialistes; nous y reviendrons.

La même Revue n'a toutefois aucune peine à s'approprier les thèses du professeur de l'Université fondée par Eugène Ruffy lorsqu'il défend des vues identiques aux siennes: ainsi la Banque d'Etat, conjointement attaquée par les libéraux pour son étatisme excessif et par les radicaux romands, très chatouilleux sur la question fédéraliste et qui apercevaient dans cet institut un hydre centralisateur inacceptable. La Revue relate en détail deux conférences que Pareto consacre à ce sujet et énumère avec délectation tous les arguments que le professeur aligne contre cette Banque imaginée par le conseiller fédéral Walter Hauser, membre du parti radical, fondé en février 1894, mais issu des rangs des démocrates zurichois, alors très marqués à gauche. «L'Etat est un mauvais banquier, comme il est un mauvais industriel et un mauvais commerçant», et, dans son inutilité et sa dangerosité, représente un péril inflationniste omniprésent ${ }^{6}$ : le réquisitoire de Pareto est sévère et réjouit des radicaux très libéraux quand il s'agit de protéger les prérogatives cantonales...

Le radicalisme a-t-il en définitive influencé Pareto? Avec Giovanni Busino on peut suggérer que Pareto a pu concevoir l'illusion que le radicalisme démocratique tendait vers les mêmes buts que lui ${ }^{7}$; et comme le rappelle le même auteur, Pareto a réellement admiré les réalisations du radicalisme ${ }^{8}$. Et sans doute a-t-il aussi lu dans le radicalisme, qui a su construire un système libéral et démocratique

4 Revue, 25.4.1893.

5 Revue, 5.2.1894.

6 Revue, 16.1 et 23.1.1897.

7 Busino, Introduction, op. cit., p. 151.

8 Giovanni Busino, La Suisse de Vilfredo Pareto, in Genève et la Suisse dans la pensée politique, Presses universitaires d'Aix-en-Provence, 2007, p. 323. 
peu fréquent à l'époque, l'avènement d'une nouvelle élite, comme il avait prêté aux socialistes la capacité de renouveler les élites fatiguées de son temps en Italie. Quand il verra que le radicalisme n'était pas exempt de dérives étatistes, notamment sur le plan fiscal et qu'il dénoncera, il reviendra toutefois vers le libéralisme démocratique, véritablement plus proche de son «aristocratisme» antiautoritaire 9 . Ces quelques remarques ne disent toutefois rien de la réalité politique vaudoise du temps où Pareto vivait à Lausanne. Les aventures des partis vaudois ont-ils pour le moins confirmé le scepticisme, pour ne pas dire plus, du savant italien envers le système parlementaire en général et les partis politiques en particulier ${ }^{10}$ ? On se saurait le prétendre. Il est cependant intéressant d'examiner le déroulement de la vie politique vaudoise durant l'époque lausannoise de Pareto, c'est-à-dire celle qu'il a eue sous les yeux entre 1893 et 1900 . C'est à cette thématique que sont consacrées les pages qui suivent.

\section{LA SITUATION POLITIQUE VAUDOISE EN 1893}

A peine Pareto a-t-il pris ses nouvelles fonctions au sein de l'Alma mater lausannoise que non seulement le petit monde politique vaudois, mais aussi l'ensemble du canton, est secoué par une sinistre nouvelle: le très populaire conseiller fédéral Louis Ruchonnet décède, le 2 septembre 1893. L'homme avait su imposer son élan d'homme d'Etat; l'ancien combattant radical prêt à ferrailler contre les ultramontains avait cédé la place, depuis son élection au gouvernement fédéral, à un politicien d'envergure supérieure qui avait compris que le champ de tension traditionnel en Suisse depuis 1848 s'était déplacé. La ligne de fracture séparait traditionnellement radicaux laïques, souvent protestants et adeptes d'un progrès économique et social, et conservateurs, en majorité catholiques et amoureux des anciennes structures fédéralistes du pays, garantes de la préservation de leurs territoires ancestraux. Elle s'était désormais effacée devant une opposition inédite, entre un libéralisme qui doit continuellement se réformer, notamment sur le plan social, et un socialisme qui exige, bien que modérément en Suisse par rapport à l'étranger, une refonte totale des institutions économiques et politiques du pays, sinon la révolution, pour les plus extrémistes.

Si cet antagonisme constitue la toile de fond des débats politiques qui animent également la vie politique vaudoise, le décès de Ruchonnet n'en est pas le catalyseur. La redistribution des cartes politiques qui est en train de s'opérer en terre

${ }^{9}$ Fiorenzo Mornati, La création de l'Ecole des sciences sociales (1890-1902). Le rôle de Vilfredo Pareto, in Olivier Meuwly et Bernard Voutat (sous la direction de), Les Constitutions vaudoises 1803-2003. Miroir des idées politiques, Lausanne, Bibliothèque historique vaudoise $\mathrm{n}^{\circ} 123$, 2003, pp. 370-375.

${ }^{10}$ Paolo Pombeni, Introduction à l'histoire des partis politiques, traduit de l'italien par Isabelle Ruchet, Paris, Presses universitaires de France, 1992, p. 173-175. Le parti radical, en général appelé le parti démocratique, est fondé officiellement en mars 1881, au moment de l'élection de Ruchonnet au Conseil fédéral, sous le nom d'Association démocratique vaudoise. Les libéraux, après une décennie 1880 douloureuse pour eux, commencent à se réorganiser dès 1890 et fondent l'Union patriotique libérale le 9 novembre 1892, à Vevey (Alain Clavien, Histoire de la Gazette de Lausanne. Le temps du colonel 1874-1917, Vevey, L'Aire, 1997, p. 122). 
vaudoise a commencé en mai 1892. Après des décennies de lutte, libéraux-conservateurs et radicaux, les ennemis habituels de la politique vaudoise, ont décidé d'enterrer la hache de guerre alors qu'entre en scène un nouvel adversaire: le socialisme. Jusqu'à maintenant, les radicaux disposaient du soutien des forces ouvrières, en général issues de la société du Grutli et dirigées par Aloys Fauquez, autour d'une Union ouvrière fondée en décembre 1890. Mais la tension monte entre les alliés. Fauquez, un ancien radical haï par ses anciens camarades de parti, avait conservé son amitié pour Ruchonnet, ce dont profitaient les radicaux. Mais, dès le début des années 90 , les problèmes s'accumulent et les Grutléens, longtemps radicaux mais de plus en plus proches du jeune parti socialiste suisse fondé en 1888, jugent désormais sévèrement l'engagement social des radicaux, qu'ils estiment insuffisant. Les radicaux s'énervent, de leur côté, des provocations grutléennes et s'obstinent à se considérer comme les seuls à même de défendre l'ouvrier et le pauvre ${ }^{11}$.

Alors que, au niveau suisse, les radicaux prennent acte de la nouvelle donne en accueillant un conservateur-catholique au Conseil fédéral en 1891, puis en s'organisant en parti politique, en 1894, pour mieux répliquer aux revendications socialistes et à celles de conservateurs pas encore à l' aise dans leur rôle de «partenaire minoritaire» au sein du gouvernement. Dans le canton de Vaud, radicaux et libéraux-conservateurs, après la rude bataille constitutionnelle de 1885, ont certes tenté un premier rapprochement, mais en vain, tant les tensions sont grandes entre les deux partis: les libéraux furent certes conviés à rejoindre le Conseil d'Etat, mais à condition que leur candidat eût reçu l'aval des radicaux... Inacceptable! L'évolution malheureuse du dossier ferroviaire, puis le divorce qui s'amorce avec les socialistes, quand bien même certains radicaux pensent encore ramener ces derniers au bercail radical au terme d'une brouille forcément passagère, changent le paysage politique ${ }^{12}$.

En 1892 éclate le scandale Vessaz, l'événement décisif qui va imprimer un nouveau cours à la politique vaudoise. Antoine Vessaz, proche ami de Ruchonnet, est un homme puissant. Successivement président du Conseil national et du Conseil des Etats, désormais receveur mais surtout l'homme clé du grand projet ferroviaire du tunnel du Simplon, il s'est attiré un nombre inouï d'inimitiés: homme craint, autoritaire, il avait amarré, avec l'aide de Ruchonnet, la compagnie romande du Suisse-Occidentale, porteuse du futur tunnel, à la compagnie de JuraBerne-Lucerne, pour mieux assurer la construction du tunnel. Mais quand il s'aperçoit que les Bernois, responsables de la compagnie fusionnée du Jura-

${ }^{11}$ L'hostilité des radicaux envers Fauquez poussa le Grutli à présenter, désormais, ses propres listes. Cette opération préluda à la création de l'Union ouvrière, le 2 décembre 1890, comme cartel syndical et groupement politique. Aux élections au Conseil national d'octobre 1890, 1'Union ouvrière soutint Fauquez et quatre des cinq candidats radicaux, Louis Paschoud, de Lutry, étant évincé. Le second tour opposa ce dernier au libéral Boiceau: Fauquez se retira en faveur du radical avec la promesse d'être présenté à la prochaine occasion. Paschoud sera élu. Or, lors d'une complémentaire de 1891, les radicaux proposeront Pernoux, le président du Grutli de Vevey! Fauquez avait été joué; il ne le pardonnera jamais à ses anciens amis (André Lasserre, La classe ouvrière dans la société vaudoise 1845 à 1914, Lausanne, Bibliothèque historique vaudoise no 48, pp. 180-181).

${ }^{12}$ Olivier Meuwly, Louis Ruchonnet 1834-1893. Un homme d'Etat entre action et idéal, Lausanne, Bibliothèque historique vaudoise no 128, 2006, pp. 388-408. 
Simplon, visent avant tout une centralisation des compagnies, sans se soucier du destin du Simplon, le sang de Vessaz ne fait qu'un tour: il manœuvre pour évincer ces si peu fiables Bernois... Mais Vessaz est aussi un homme d'argent. Peu après, le journal radical bernois Der Bund révèle l'impensable: Vessaz a touché des potsde-vin de financiers allemands au moment de la création du Jura-Simplon!

Dans le canton de Vaud, c'est le choc. Vessaz doit quitter avec effet immédiat tous ses mandats et les libéraux, victimes récurrentes de l'autoritarisme du rusé receveur, jubilent: Vessaz n'incarnait-il pas l'arrogance des radicaux qui, durant la décennie 1880, avait occupé sans vergogne tous les postes en affichant leur mépris souverain de l'opposition? Ils se refusent toutefois à lancer une offensive sanglante contre leurs adversaires. Le climat politique rappelé ci-dessus ne permet plus d'imprudentes rancunes. Habilement, l'influent député Louis Berdez propose aux radicaux la paix des braves. Exclus depuis onze ans des affaires, ayant refusé d'accepter un strapontin gouvernemental à n'importe quel prix, ils offrent leurs services, au nom d'une politique apaisée et ouverte aux principales forces politiques du canton. Non moins habilement, Eugène Ruffy, chef des radicaux avant de succéder à Ruchonnet en 1893 au Conseil fédéral, mais sans doute avec l'accord au moins tacite de celui-ci, comprend le message: à la première occasion, un siège sera laissé aux libéraux au Conseil national et au Conseil d'Etat. C'est le compromis dit du 6 mai 1892.

\section{LA MISE EN OEUVRE DU COMPROMIS DU 6 MAI}

L'épreuve de vérité survient en janvier 1893. Le 8 janvier décède le conseiller national Paschoud, un autre fidèle de Ruchonnet. Les radicaux de l'arrondissement regroupant le Chablais, Lavaux et Lausanne se réunissent comme de coutume à Chillon pour arrêter leur stratégie, sous la présidence d'Adolphe Dupraz, ancien député et président de la société électrique de Vevey-Montreux ${ }^{13}$. Eugène Ruffy, en tant que président de l'Association démocratique défend le compromis, rappelle qu' «une parole loyalement donnée doit être loyalement tenue». Ruffy entend couper l'herbe sous les pieds de certains radicaux qui lorgnaient sur le siège laissé vacant et, surtout, sur les prétentions d'autres radicaux désireux d'abandonner ce siège au parti ouvrier. Sur ce dernier point, Ruffy se veut limpide: à ses yeux, «le parti démocratique a toujours accueilli avec sympathie les revendications ouvrières d'un caractère juste et équitable», il «n'a pas attendu, pour s'occuper des réformes sociales, qu' on lui forçât la main et si la Suisse a devancé les autres nations dans cette voie, (il) peut à bon droit s'en attribuer le mérite». Et le futur conseiller fédéral d'énumérer les grandes victoires radicales, comme la loi sur les fabriques, la responsabilité des entrepreneurs ou l'assurance obligatoire, alors en chantier, en cas de maladie et d'accident.

Mais l'opération se heurte à des obstacles inattendus. Certains estiment légitime que, si les radicaux acceptent de tendre la main aux libéraux, ils puissent au moins se prononcer sur le nom du candidat conservateur. Les radicaux avaient torpillé diverses démarches de réconciliation dans les années 1880 avec des

${ }^{13}$ Revue, 6.2.1893. 
manœuvres similaires, on s'en souvient... Ces radicaux redoutent surtout le retour de l'ancien conseiller fédéral Paul Cérésole, l'un des libéraux les plus virulents, un homme avec lequel ils ont souvent eu maille à partir, un homme qui suscite une animosité réelle dans les rangs radicaux. Leurs préférences vont vers le sage Charles Boiceau, quand bien même il avait quitté le Conseil national avec ses deux collègues libéraux en 1881, en signe de protestation contre la volonté des radicaux de détenir tous les sièges à la Chambre basse du Parlement fédéral. L'assemblée radicale veut un compromis sans Cérésole et le fait savoir, par 103 voix contre 69 ! Ruffy s'en sortira en disant que si les libéraux choisissaient quelqu'un d'autre, les radicaux s'abstiendraient de désigner un contradicteur. C'est bien vu: les libéraux plébiscitent Cérésole. Au nom de la nécessaire réconciliation, les radicaux adoptent un profil bas...

La Revue regrette le choix de ces décidément encombrants alliés et, au soir de l'élection, ne manquera pas de se moquer de la victoire tout sauf imposante de Cérésole contre le chef ouvrier, Aloys Fauquez ${ }^{14}$ : «Le candidat ouvrier a bénéficié de l'erreur de tactique des conservateurs », grâce à une forte abstention du côté des radicaux. Puis la polémique glisse vers la presse où, contre la Revue, ferraille le Nouvelliste vaudois, l'ancienne feuille officielle des radicaux et, désormais, l'organe le plus combatif des libéraux. En répliquant tout aussi durement aux attaques du Nouvelliste, la Revue peut fustiger une aile des libéraux qu'elle regroupe au sein d'un indistinct «parti des actionnaires», des «mômiers » et des «aristocrates entichés de leur nom et de leurs origines », sans tonner directement contre les libéraux-conservateurs dans leur ensemble: la stratégie d'alliance est sauve...

Car tout faux pas est interdit, d'autant que se profilent déjà les élections cantonales du printemps 1893. A Lausanne, le comité démocratique, porte-parole des radicaux du chef-lieu, prend langue avec les conservateurs et les ouvriers, afin de «provoquer, si possible, une entente entre les partis dont l'existence à Lausanne est consacrée par les faits ». La démarche est habile: contre socialistes et libéraux qui réclament l'introduction de la représentation proportionnelle, les radicaux, qui s'y opposent avec la dernière énergie, tentent d'adoucir les humeurs vindicatives de leurs concurrents en assurant une judicieuse répartition du pouvoir qui n'écornerait pas trop le leur... Les conservateurs répondent favorablement aux avances radicales mais l'Union ouvrière annonce ne pouvoir y accéder qu'à une double condition: que des ouvriers soient soutenus dans les autres cercles où ils se présenteraient et qu'une liste d'entente soit également élaborée pour les élections fédérales de l'automne 1893.

Les dirigeants des trois partis se rencontrent le 24 février, avant de se séparer sur un constat de désaccord ${ }^{15}$. Les demandes ouvrières sont jugées irrecevables; les radicaux et les libéraux partiront seuls au combat. Mais l'entente, en bonne

${ }^{14}$ Revue, 8.2, 13.2 et 14.2.1893.

${ }^{15}$ Revue, 27.2.1893. Pour les radicaux sont présents, Marc Ruchet, ancien stagiaire de Ruchonnet, futur successeur de Ruffy au Conseil d'Etat et au Conseil fédéral, Emile Paccaud, un ami de longue date de Ruchonnet et l'un des radicaux les plus puissants du canton, et le receveur de Kaenel; pour les libéraux, Adrien Demiéville, Eugène Francillon et Edouard Secrétan, le rédacteur en chef de la Gazette de Lausanne; pour les ouvriers, Aloys Fauquez, le cordonnier François Kaufmann, président de l'Union ouvrière, et le typographe Blanc. 
voie à Lausanne, connaît aussi des ratés : les plaintes se multiplient; dans plusieurs cercles, d'aucuns estiment que le compromis du 6 mai n'est pas appliqué avec la loyauté requise ${ }^{16}$. La Revue minimise les brouilles locales: n'est-il pas bien que des luttes demeurent dans certains cercles? Le compromis du 6 mai ne doit-il pas être compris dans son esprit plutôt qu'au pied de la lettre? Des concessions n'ontelle pas été faites lors d'élections partielles? Et ne faut-il pas aussi éviter un trop fort émiettement, sous-entendu de la force radicale, alors que les minorités ont été de tout temps représentées? Le pacte, à Lausanne, est respecté et, d'un commun accord et sans la ratification des dirigeants des travailleurs, des places réservées aux candidats ouvriers pour le second tour au cas où aucun d'entre eux ne passerait au premier tour. Les radicaux ne veulent en rien renoncer à la pratique de la «proportionnelle volontaire», qui apaise les oppositions...

Les radicaux lausannois se lancent dans la bataille l'esprit serein. Sous la présidence de Ruchet, ils tentent de bétonner leurs positions, surtout face aux reproches de la gauche ${ }^{17}$. Alors que cette dernière attaque leur immobilisme, ils égrainent leurs réalisations: constructions nouvelles, institution cantonale pour l'enfance malheureuse, nouveaux établissements hospitaliers, l'Université, l'école primaire, la formation agricole. Les radicaux se sont rendus à l'évidence: les dissidents ouvriers ne reviendront jamais dans leur giron. Sans doute Ruchet évoque-t-il encore «d'anciens amis politiques séparés de nous, momentanément, je l'espère »; sans doute martèle-t-il que «cette avant-garde nous reviendra parce qu'elle a les mêmes aspirations que nous et qu'il nous faut travailler tous ensemble si nous voulons voir aboutir les réformes souhaitées ». Pourtant, la polémique prend un tour aigre et révèle un fossé infranchissable. Les socialistes exigent-ils le progrès? Ruchet s'en défend: "Cette idée, c'est la nôtre, nous l'avons tous dans le cœur», affirme-t-il, sous les applaudissements de son auditoire. De fait, le réformisme radical s'allie naturellement avec le conservatisme des libéraux: l'alliance électorale n'est qu'une formalité, y compris l'abandon de deux sièges aux ouvriers. L'animosité socialo-radicale alimente l'amitié libéraloradicale, au-delà des clivages qui déchirent encore les deux anciens ennemis. Les ouvriers ont beau tempêter, accabler les «tripotages » des radicaux et libéraux, rappeler la promesse de 1889, hurler à l'hypocrisie, à l'injustice: «Jamais on ne vit, dans le canton de Vaud, pareils marchandages politiques, semblable escamotage du suffrage populaire (...) Ceux qui, en 1885, se menaçaient d'entrelardement réciproque, se donnèrent le baiser de Judas ${ }^{18}$. L'obstacle idéologique, masqué par les querelles personnelles, a redessiné les camps en présence.

Les élections de mars ne provoquent aucun renversement au sein du Grand Conseil. A 65 conservateurs faisaient face 145 radicaux environ: le nouveau Parlement offrira un visage quasiment identique. A Lausanne, après le premier tour, 16 des 25 sièges sont repourvus: selon l'accord survenu entre libéraux et radicaux, la liste commune portera les noms de cinq conservateurs, deux radicaux, celui du président de l'Union ouvrière Kaufmann et une ligne blanche, réservée à un candidat socialiste. Les radicaux souhaitent que le «malentendu» avec l'Union

\footnotetext{
16 Revue, 1.3.1893.

17 Revue, 3.3.1893.

${ }^{18}$ Grutli, 10.2 et 10.3 .1893 .
} 
ouvrière soit bientôt résorbé, mais l'affiche ouvrière, très violente envers les radicaux, a mis les nerfs à vif... Les électeurs lausannois suivent la consigne des deux principaux partis, en offrant un beau succès à Kaufmann, qu'ils flanquent de Fauquez. La bonne ambiance qui règne entre libéraux et radicaux se confirme lors du renouvellement du Conseil d'Etat. Comme convenu un an auparavant, un siège gouvernemental doit être laissé aux libéraux. Or, miraculeusement, le radical Donat Golaz annonce son retrait. A-t-il été poussé vers la sortie, avec la promesse d'un poste de receveur du district de Vevey ${ }^{19}$ ? Les libéraux sautent sur l'occasion et ont l'intelligence de présenter un candidat au profil moins acéré que Cérésole. Ils se coalisent derrière l'avocat Robert Cossy, dont la désignation est saluée avec joie par la Revue: «Si l'on veut une conciliation sincère et durable, c'est avec le concours d'hommes loyaux et de bonne volonté qu'on la réalisera $»^{20}$. Pour les radicaux, nul doute que l'impétrant appartient à cette catégorie de personnes.

\section{LES AFFRES DE LA SUCCESSION DE RUFFY}

Pour les radicaux, il n'existe en effet plus qu' une issue. Si le retour des dissidents ouvriers est souhaitable, l'avenir se dessine avec les libéraux. Les radicaux tentent de ne pas baisser la garde sur le plan social, et se rendent compte que, pour ambitieuse que leur politique en la matière paraisse, elle ne peut plus convaincre un monde ouvrier qui n'envisage plus aucune rédemption du côté bourgeois. Ils conservent néanmoins leur objectif supérieur, consistant à fédérer toutes les couches de la société en un parti national, dans lequel la réconciliation entre classes s'opérerait naturellement. L'alliance avec le grand capital zurichois, les conservateurs catholiques et les conservateurs protestants n'altère en rien, dans leur esprit, le progrès social. Mais ils ne sont pas à l'abri de redoutables contradictions!

Au niveau fédéral, ils misent sur la loi sur l'assurance contre la maladie et les accidents, dont la mise en œuvre législative est alors à l'étude, sous la conduite du démocrate zurichois et futur conseiller fédéral Ludwig Forrer. Mais la tension entre ouverture sociale et respect d'une stricte orthodoxie financière minera leurs efforts, et achèvera de déconsidérer le radicalisme hégémonique aux yeux des ouvriers. Les radicaux continuent néanmoins à se positionner au centre de la synthèse sociale que les temps appellent. La Revue ne manque pas de rappeler régulièrement les fruits du labeur radical, dans l'espoir d'endiguer le flot des

${ }_{19}$ Pierre-André Bovard, Le gouvernement vaudois, Morges de 1803 à 1962. Récit et portraits, Morges, Editions de Peyrollaz, 1962, p. 197. La Revue présente la démission de Golaz comme ayant été conditionnée par l'impopularité, qu'il s'était attirée à la suite de la réorganisation de l'économat de l'Etat qu'il avait impulsée, et par les attaques dont il avait été la cible en luttant contre les empiétements systématiques de Berne... L'argument paraît en effet spécieux quand on connaît l'esprit fédéraliste des radicaux vaudois, un esprit qui s'était certes adouci avec l'accession de Ruchonnet au Conseil fédéral mais qui restait néanmoins vif. Relayant le Journal de Genève qui brocarde la capacité de Golaz à se mettre tout le monde à dos par son esprit borné, le Grutli affirme que le Conseil d'Etat renoncera à proposer leur collègue pour le poste envisagé sous la pression de l'opinion publique (Grutli, 7.4.1893).

${ }^{20}$ Revue, 23.3.1893. 
revendications socialistes: imposition directe la plus forte de Suisse, incinérations gratuites, tribunal des prud'hommes. Pour elle, ce travail ne peut cependant aboutir que si les autorités ne sont «pas abreuvées d'outrages, d'injures et de gros mots par certains hommes qui prétendent au monopole du progrès social $» . .{ }^{21} \mathrm{Et}$ c'est l'une des cibles privilégiées du Grutli, Emile Paccaud, l'homme "aux 20 '000 francs» accusé de cumuler les prébendes, d'être «le gouverneur du gouvernement $»^{22}$, actif autant au Conseil communal de Lausanne qu'au Grand Conseil, qui défend, à Lausanne, une pétition radicale demandant la création par la Municipalité d'une caisse d'assurance contre le chômage, à l'instar de ce qui existe déjà à Berne, notamment. De son côté, la Revue s'étend sur le principe de la solidarité qui doit, par l'association coopérative, promouvoir l'aide en complément de l'Etat, reconnu «comme une forme très remarquable, la forme le plus large, de la solidarité $»^{23}$.

Ce nouvel équilibre gauche-droite en train de se matérialiser n'a cependant pas épongé toutes les vieilles rancœurs. L'aile droite des libéraux et l'aile gauche des radicaux ne se satisfont pas de la nouvelle stratégie d'alliances, rêvent encore de pouvoir en découdre avec l'ennemi héréditaire... Le conflit resurgit à l'occasion d'une élection complémentaire au Grand Conseil à Lausanne, pour remplacer Eugène Ruffy, réélu au Conseil d'Etat. Les radicaux estiment normal que l'un des leurs succède à l'élu, ou au moins que le siège soit laissé au parti ouvrier. Ils désignent à cet effet Paul Vulliet, un ancien professeur de l'Ecole industrielle, très respecté par les ouvriers. Mais les conservateurs réclament le siège pour euxmêmes! Sentant l'«alliance clérico-aristocratico-momico-radicalo-démocraticodoctrinaire» se fissurer, la gauche saute sur l'occasion et envoie au combat son champion, l'avocat Paul Panchaud, qui l'emportera au second tour, alors que les radicaux avaient retiré leur candidat, défait dès la première ronde ${ }^{24}$.

Les organes de presse des deux grands partis entrent dans une profonde fureur. La Revue énumère les concessions faites aux libéraux, rappelle que leur chef de file Berdez a reçu nombre de gros procès impliquant l'Etat, tandis que la Gazette accuse les radicaux de trahison. Le renouvellement complet de la députation vaudoise aux Chambres fédérales de l'automne s'annonce mal. Pour l'arrondissement de Lausanne-Lavaux-Chablais, les libéraux, sous la présidence d'Aloys de Meuron, reconduisent néanmoins l'alliance, mais sans enthousiasme, par 88 voix contre $68^{25}$. Par la voix de leur président Ruffy, les radicaux confirment à leur tour leur engagement antérieur et laissent la place abandonnée par Pernoux à un conservateur, Charles Boiceau, l'exclu du début de l'année. Les discours les plus virulents sont en réalité adressés aux représentants des ouvriers. Condamnant l'attitude de la feuille ouvrière, Ruffy vise leur loyauté, fouillant dans leur programme une reproduction lénifiante des exigences beaucoup plus agressives

${ }^{21}$ Revue, 11.4 et 12.4 .1893 .

${ }^{22}$ Grutli, 14.4.1893 et 11.1.1894. Les députés ouvriers déposeront en avril 1893 une motion visant à interdire le cumul des mandats de député et de directeur de la Banque cantonale (poste qu'occupait Paccaud) ou du Crédit hypothécaire.

${ }^{23}$ Revue, $10.10,11.10$ et 12.10 .1893 .

${ }^{24}$ Grutli, 21.4.1893.

${ }^{25}$ Clavien, Histoire de la Gazette de Lausanne, op. cit., p.118. 
émises par un congrès socialiste international, qui s'était tenu à Zurich quelques mois auparavant. Pour Ruffy, d'éventuels pourparlers avec les ouvriers sont désormais impensables: «La fraction des ouvriers condamne la propriété individuelle, sacrifie la liberté, menace toutes les conquêtes de la liberté et traite les progressistes comme aucun parti réactionnaire ne l'a fait jusqu'ici ${ }^{26}$. Paul Vulliet, le vaincu d'avril, tente néanmoins de convaincre l'assemblée radicale de Chillon de laisser une place libre aux «ouvriers-socialistes», mais son appel ne passe pas: les radicaux n'apprécient guère ce qu'ils considèrent comme un noyautage de la salle par quelques ouvriers organisés, à la manière des sociaux-démocrates allemands... ${ }^{27} \mathrm{Sa}$ proposition est balayée par 320 suffrages contre 25 . Le Grutli est dépité: «Le comité doctrinaire s'allie ouvertement aux rétrogrades contre le parti avancé », conclut-il ${ }^{28}$. Quant aux ultimes élections de cette lourde année 1893, les élections communales, elles soulignent sans frottement la domination radicalo-libérale. Mais l'affaire de la succession de Ruffy a laissé des traces.

\section{VERS LA RÉCONCILIATION DÉFINITIVE ENTRE RADICAUX ET LIBÉRAUX}

Les engagements prononcés en 1893 semblent oubliés. Lors de l'élection complémentaire au Conseil national destinée à remplacer Ruffy réélu au Conseil fédéral, les radicaux proposent logiquement un candidat, en la personne de l'un de leurs chefs, le Veveysan Emile Gaudard, un ancien libéral d'ailleurs. Mais les libéraux refusent de reconnaître cette sorte de succession automatique et revendiquent à nouveau le siège! Pire encore pour les radicaux: après avoir refusé de laisser la voie libre au candidat radical et écarté le modéré Berdez, leur assemblée désigne l'homme le moins à même de complaire à leurs alliés, le colonel et rédacteur de la Gazette de Lausanne, Edouard Secrétan. La campagne recouvre dès lors les accents des luttes des années 1880: la Revue dénonce la «politique à courtes vues » des libéraux et clame son hostilité au candidat conservateur en titrant qu'il y a «trop de colonels » à Berne! Au soir du premier tour, Gaudard mène devant Secrétan avec 1'700 voix environ d'avance, et Fauquez, qui en récolte 2'500. Au second tour, Gaudard l'emporte largement, bénéficiant certes de bon report des voix socialistes $^{29}$, mais aussi du score plus que mitigé que Secrétan récolte auprès de ses propres troupes. Le vaincu s'en émeut, auprès de son ami neuchâtelois Philippe Godet: il reconnaît, amer, que 1'000 à 1'200 libéraux ont renoncé à le soutenir $^{30}$. Si l'alliance est encore fragile, comme on le verra encore, les libéraux modérés semblent prendre le dessus.

${ }^{26}$ Revue, 23.10.1893.

${ }^{27}$ Paul Vulliet, membre de la société du Grutli comme tous les ténors radicaux, sera élu au Grand Conseil en juin 1896 sur une liste radicale, mais avec le soutien des ouvriers (Claude Cantini, Le Grütli (1890-1909). Miroir du socialisme vaudois naissant, in Les origines du socialisme en Suisse romande 1880-1920, Association pour l'étude de l'histoire du mouvement ouvrier, cahier $\mathrm{n}^{\circ}$ 5/1988, p. 49).

${ }^{28}$ Grutli, 20.10.1893.

${ }^{29}$ Revue, 15.1, 16.1, 22.1 et 24.1.1894.

${ }^{30}$ Clavien, Histoire de la Gazette de Lausanne, op. cit., pp. 125-126. 
Dans ces conditions, il n'est néanmoins pas étonnant que la fondation à Olten du parti radical-démocratique suisse, fruit de la fusion entre les radicaux romands, les démocrates alémaniques et la quasi-totalité des libéraux alémaniques, laisse froids les Vaudois. Il est vrai que, comme à Genève, Bâle-Ville et Neuchâtel, les libéraux représentent réellement le camp conservateur, ce qui était moins le cas dans la plupart des cantons alémaniques. Mais il n'empêche: l'ambiance qui règne sur les bords du Léman ne favorise guère un commerce courtois entre les deux partenaires. Calmement, la Revue explique l'impossibilité de répéter dans le canton de Vaud l'opération, qu'elle salue par ailleurs, en train d'être conclue outre-Sarine: «Le parti progressiste suisse a pris conscience de son unité indépendante de divergences inévitables dans un pays composé comme le nôtre, où la géographie, l'histoire, les traditions ont semé comme à plaisir les particularités régionales ${ }^{31}$. Les élections cantonales partielles confirmeront le rafraîchissement des relations entre radicaux et libéraux vaudois.

Quelques jours avant la création du nouveau parti, une triple partielle avait débouché sur une débâcle libéralo-radicale, qui avait vu l'Union ouvrière faire passer ses trois candidats: le garde-frontière Adolphe Gavillet, le typographe Jules-Félix Blanc et Charles Peneveyre ${ }^{32}$. Ce ne fut guère mieux lors des élections communales de l'automne, où Fauquez est accusé d'avoir soutenu les listes conservatrices contre les radicaux ${ }^{33}$. Et l'année 1894 ne débute pas sous de meilleurs auspices. En août, alors que le radical lausannois Piot vient de décéder, les libéraux renoncent certes à réclamer le siège, au nom de «l'entente globale avec le parti radical », mais, à la grande colère de la Revue, se contente de prêcher l'abstention ${ }^{34}$. Ennui : les radicaux n'ont pas de candidat à disposition et pensaient que les libéraux se lanceraient... Le résultat de cette cacophonie ne se fait pas attendre: est élu un nouveau socialiste, le postier Jules Couchepin. Pour la feuille radicale, la cause est réglée: socialistes et libéraux ont communié dans une alliance tacite, alors que les deux formations exigeaient depuis longtemps le vote proportionnel. Une nouvelle partielle, à la suite du décès d'un radical lausannois, en octobre de la même année, poussera-t-elle les alliés à un nouvel affrontement larvé? Or, cette fois, les deux partis semblent entendre la partie de «l'opinion publique (qui) demande que l'entente se fasse et que les partis non socialistes réunissent leurs efforts pour affirmer leur majorité $»^{35}$. Dans la Revue, le comité radical relève que «les suites d'une rupture persistante entre les deux partis et la continuation des victoires socialistes auraient des résultats déplorables pour les

${ }^{31}$ Revue, 27.2.1894. Sur ces questions, voir aussi Olivier Meuwly, L'unité impossible. Le parti radical-démocratique suisse à la Belle Epoque 1891-1914, Hauterive, Gilles Attinger, 2007.

32 Grutli, 23.2.1894.

${ }^{33}$ Plus tard, Félix Bonjour cherchera à minimiser les tensions surgies entre radicaux et libéraux. Pour l'élection du 16 avril, qui avait vu la victoire de Panchaud, il estimera que «l'entente fut un peu troublée» à l'occasion de cette élection... Bel euphémisme qu'il justifiera justement en attribuant la responsabilité de la défaite aux menées de Fauquez (Félix Bonjour, Souvenirs d'un journaliste, tome I, Lausanne, Payot, p. 154). La haine que vouent les radicaux à leur ancien ami est un élément central, mais pas déterminant, de la vie politique de cette période.

${ }^{34}$ Revue, 4.8.1894.

${ }^{35}$ Revue, 5.10, 6.10 et 15.10.1894. 
intérêts de la ville de Lausanne et sa situation devant le canton »; cette capitale à la forte population ouvrière qui tourne si dangereusement au rouge... L'entente se solde par un succès: l'avocat Dubuis est élu, en dépit d'un beau score de son rival de gauche. La Revue s'empressera de louer la loyauté de la majorité des conservateurs, pour mieux égratigner les plus intransigeants, retranchés dans les colonnes du Nouvelliste! Ou est-ce une victoire à la Pyrrhus, comme le suggère le Grutli $^{36}$ ?

Alors que le journal ouvrier assène qu'il n'existe plus de radicaux, que ces «larbins » de «Ruffy-Manitou» ne savent que s'adonner à «l'amour des titres, des hochets, et (à) l'ambition primant l'intérêt général ${ }^{37}$, que «le parti socialiste était le seul parti d'opposition ${ }^{38}$, les élections partielles suivantes se passent sans heurt. Même état d'esprit lors des élections nationales de 1896. Malgré les efforts de l'infatigable Paul Vulliet, qui souhaite toujours qu'une place soit réservée à un candidat ouvrier, qui supplie d'oublier la violence des journaux, les radicaux restent inflexibles et rejettent la proposition de leur collègue ${ }^{39}$. La Revue insiste néanmoins, souligne que le fossé entre radicaux et gauche est désormais infranchissable et veut appeler le parti ouvrier par son vrai nom, le «parti socialiste ${ }^{40}$. Mais elle ne pourra s'empêcher de morigéner les libéraux en découvrant le piètre résultat de Cérésole, réélu mais battu dans sa ville de Vevey: les libéraux ont besoin des radicaux, insinue-t-elle, qu'ils y pensent quand il s'agira de s'arranger sur l'emplacement du futur Palais de Rumine...

L'entente revenue éclairera les deux partis bourgeois sur la réalité sociologique de la capitale vaudoise. Hostiles à la proportionnelle, les radicaux lausannois proposent pourtant aux libéraux et aux socialistes, à l'occasion des élections cantonales du printemps 1897, un compromis, en vain. Une proportionnelle de fait sera toutefois instituée, favorisant... les radicaux, qui confortent leur position, malgré la création d'un parti progressiste, formé en réalité de jeunes radicaux et qui raflera un siège ${ }^{41}$. Le nouveau Grand Conseil opposera désormais aux 140 radicaux et 60 libéraux, en chiffres ronds, 11 socialistes, dont Oscar Rapin. En revanche, aux élections communales de l'automne, un arrangement est conclu pour le second tour, mais résistera mal à la réalité politique. Avec une Municipalité dominée par les conservateurs mais complètement discréditée, la tension est à vif: les socialistes ont 39 élus, les radicaux 31, les libéraux, en chute libre, 24, alors que ses deux municipaux restent sur le carreau, et les progressistes $6^{42}$. La gauche se sent le droit de revendiquer la syndicature, que lui refusent les bourgeois, qui réélisent comme syndic le libéral van Muyden, désavoué devant le

${ }^{36}$ Grutli, 19.10.1894.

37 Grutli, 13.3.1896.

${ }^{38}$ Grutli, 12.5.1899.

${ }^{39}$ Revue, $19.10,20.10$ et 26.10.1896.

${ }^{40} \mathrm{Il}$ semble que le Grutli intitule pour la première fois le parti qui lui est proche «parti socialiste » en 1899 (Cantini, Le Grütli (1890-1909), art. cit., p. 43).

${ }^{41}$ Revue, 15.1, 23.1, 5.2, 2.3 et 8.3.1897. Sans surprise, sentant le germe d'une nouvelle division chez ses adversaires, le Grutli saluera la naissance du parti progressiste qui, à ses yeux, montre le réveil des jeunes face à d' «anciens partis en décomposition » (Grutli, 5.2.1897).

${ }^{42}$ Revue, 24.11, 27.11, 30.11, 1.12 et 23.12.1897. 
peuple... Ulcérés, les socialistes renoncent alors à siéger à l'exécutif de la ville et laissent un radical, en la personne de Louis Gagnaux, un ancien radical dissident revenu en grâce, s'emparer de la plus haute magistrature !

Sur le plan cantonal, l'entente radicale-libérale se consolidera au fil des années, sous la menace de plus en plus pressante des socialistes. L'alliance pour les élections nationales est inébranlable et les radicaux balaient une nouvelle proposition de Vulliet de s'ouvrir aux socialistes: excédés, les chefs du parti lui reprochent à nouveau de se faire accompagner par des personnes non membres du parti $^{43}$. Pour Ruchet, le parti radical a autant le droit de se revendiquer ouvrier: «Ouvriers, nous le sommes tous ou nous l'avons été. Nous avons travaillé et souvent plus de huit heures par jour»... Dépendant de leur allié, les libéraux accepteront toutes les couleuvres que leur fait avaler la direction radicale: le Conseil des Etats leur est fermé et un deuxième siège au Conseil d'Etat, malgré leurs demandes insistantes, malgré la mauvaise foi dont ils accusent le parti dominant, ne leur sera accordé que dans le contexte particulier de la Première Guerre mondiale.

\section{CONCLUSION}

Pour faire barrage à l'avancée socialiste dans les centres urbains, les radicaux et les libéraux ont ainsi amorcé un rapprochement qui, en dépit de multiples avanies, s'affermira au cours des années. Le Grutli aura beau pointer la trahison présumée des radicaux, qui ont préféré ouvrir leurs bras à leurs ennemis conservateurs, le cours de l'histoire est tracé: contre un socialisme qui tend à adopter des caractéristiques de plus en plus marxisantes, l'alliance bourgeoise paraît inévitable. Pareto, dont la leçon politique, d'après Raymond Aron, est par essence ambiguë $^{44}$, a-t-il suivi les batailles politiques dans son canton d'accueil? En a-t-il tiré des analyses qui auraient contribué à alimenter sa perception globale des partis politiques? On l'a dit, son observation de la montée de l'étatisme sous la houlette des radicaux puis des socialistes, de plus en plus puissants, l'aura confirmé dans sa méfiance envers la démocratie. Le rapprochement entre libéraux et radicaux à l'œuvre sous ses yeux l'aurait-il rassuré ? On peut en douter: comme Fiorenzo Mornati l'a montré, il illustre plutôt le déclin de l'idée du libéralisme tel qu'il avait rêvé... ${ }^{45}$

\footnotetext{
${ }^{43}$ Revue, 23.10.1899.

${ }^{44}$ Raymond Aron, Les étapes de la pensée sociologique, Paris, Tel Gallimard, 1967, p. 479.

${ }^{45}$ Mornati, La création de l'Ecole des sciences sociales, op. cit., pp. 370-375.
} 